\title{
Interactive Digital Storytelling based Educational Games: Formalise, Author, Play, Educate and Enjoy! - The edugames4all Project Framework
}

\author{
Andreea Molnar ${ }^{1}$ and Patty Kostkova ${ }^{2}$ \\ 1 University of Portsmouth, andreea.molnar@port.ac.uk \\ 2 University College London, p.kostkova@ucl.ac.uk
}

\begin{abstract}
In this paper we present an Interactive Digital Storytelling (IDS) formalisation that captures the IDS logic constraints. This was implemented in a game engine and an authoring tool was created to facilitate the development of IDS based games. We created several IDS based games using the proposed engine and authoring tool. They were used as case studies in this research. We also present some of the challenges we encountered once the games were implemented and how we addressed them.
\end{abstract}

\section{Introduction}

In recent years, Interactive Digital Storytelling (IDS) based games have been growing in popularity [1] and recognised as providing a motivating and engaging experience for players [2, 3. Moreover, when storytelling is integrated in the game, it has been shown to help players make sense of their experience, improve their problem solving abilities, and ability to organise knowledge [2]. Due to their dynamical nature, they also provide the player with a better feeling of being able to control the game and how the story unfolds.

However, the design of interactive digital storytelling (IDS) 4 4 in particular, and of games in general [5] is increasingly complex. One of the challenges faced consists of a lack of "development of formalism supporting narrative description" 6]. As game story writers most often do not come from a computing background there is a need for both a logic design formalism for IDS based games and authoring tools to facilitate IDS creation. The first part of this research provides a solution to this problem by providing a platform that includes a Finite State Machine based formalisation of the IDS. The formalism is an important step in achieving a shared understanding of the field as it establishes shared requirements that can be used by future researchers. It can also be a step forward towards a fully automatic approach. The proposed formalism was used to develop a game engine.

This paper also presents the authoring tool that enables easier development of IDS based games. Furthermore, in this research we also investigated three 
challenges we encountered once the games were implemented: high drop-out rate (despite a user centric design being followed through the implementation), usability and educational content assessment issues. We present our proposed solutions to this problems.

The rest of the paper is organised as follows. Section 2 presents the state of the art in this area and pins down where this research stands. The IDS FSM formalisation is defined in Section 3 . Section 4 introduces the game engine implementation and how it connects with the authoring tool. It also presents examples of implementations using the authoring tool and a brief introduction into the edugames4all project. The following section presents the challenges encountered when evaluating the games. The next section discusses the results of this research and presents the work in progress. The paper ends with the conclusions presented in Section 7.

\section{Related Work}

The work presented in this paper spans across the following research domains: usage of artificial intelligence (AI) techniques in interactive digital storytelling, usage of interactive storytelling in educational games and educational content evaluation in educational games.

\subsection{Artificial Intelligence Techniques in Interactive Digital Storytelling}

IDS is dynamic and allows players to act as they are the main characters in the story, to select among multiple paths, and basically to modify the story based on their actions. Despite the potential of a formalisation to "allow the deduction of results which throw a specific light at research applications" [7, there is little research available in this domain on the development of a formalization for the narrative description [6].

AI techniques have been used in IDS mostly to adapt the narratives to user's actions 8 or in the implementation of different aspects of storytelling generation. Among these techniques one can count case based reasoning [9], genetic algorithms [10, goal net and fuzzy cognitive maps [11, natural language processing [12, search algorithms [13], and FSM [14]. FSMs have been increasingly used for modeling dynamic systems 14 and in this research we use them for the formalisation of the IDS.

The research done so far on formalising IDS was based on logical reasoning [6, [15] and constraints [16. Constraint planning aims to generate a good or optimal solution given a set of possible goals [17. Our aim is not to determine the optimal but to allow users the possibility of exploring as many solutions as they want as long as given the path they are taking there is a way to reach a resolution. Although many aspects of the IDS can be formalised through logical reasoning and constrains these are not necessarily suitable for all aspects of IDS 
as shown by [7. We propose to add to the state of the art in the field and to use an AI based approach formalisation based on Finite State Machines (FSM).

FSM allows an expressive representation and it allows modelling the IDS states (FSM states) and user actions (the transition function). It allows separation between the states and the transition function that could lead to the implementation of an authoring tool where the game designers could define the game states and the transition function could be implemented separately from the game engine. This allows the generation of games that make use of interactive storytelling without the game designers needing to get into the game engine code. The formalism allows to create a sequence of causal events, recombine events in the story and determine whether a sequence of events leads towards a resolution of the story.

\subsection{Usage of Storytelling in Educational Games}

Digital storytelling is said to help in acquiring knowledge, improving media literacy and user attitude and behaviour [18. When used in educational games it provides a "gateway to immersive experience" [19. It has been shown to engage players [2, improve the learning experience by allowing the players "to make sense of experience, organise knowledge, sparking problem-solving skills and increase motivation" 22. The story provides a context to learning [20, while the interaction actively involves the players in the building of the story [2. This stimulates imagination and curiosity and leads to unintentional learning [2]. Despite their educational potential [21, 22] most studies have been focused on the simulation aspect neglecting the storytelling [23. This study addresses this gap by focusing on the storytelling aspect of educational games.

\subsection{Evaluation of the Educational Content Integrated in Games}

Assessment is an essential part of any educational experience. Traditionally, assessment of learning has taken place outside of the actual game. Pre and post-play assessments of knowledge remove the task of assessment from the educational game itself and allows for a summating assessment of player knowledge and/or attitude change. Although the evaluation is considered to be one of the most important parts in educational games [24, little research has been dedicated to seamlessly integrating the assessment in the game flow. Most of the assessment is done as a separate part of the educational game, or when integrated it is often done in an intrusive manner affecting the game flow and the player experience 25]. Among the studies that proposed an assessment integrated as part of the game play one can count: [26], 27, [28, [29] and [30].

[26] integrate multiple choice questions as a part of a point-and-click gamelike simulation. The player is provided feedback if the answer provided is wrong. The game was assessed in terms of the educational potential, but the integrated assessment and the way it was perceived by students was not evaluated. 
[27] propose a similar approach to [26] in which questions are being asked in a simulation like game. The assessment is designed to be as much as possible similar to the traditional pen and paper alternative, and the students get feedback only at the end of the evaluation. In our research, we want to have the assessment integrated as seamlessly as possible in the game flow so that the player immersion is not affected. Moreover, our proposed approach allows for feedback to be provided immediately after the assessment, if desired, as this has the potential to improve learning achievements [31, 32.

EducaMovil [28] is a suite of open source mobile learning games, through which snippets of educational content and quizzes are integrated. A certain action of the game triggers a certain lesson to open, and a question is given to the learner after the educational content snippet is shown. Feedback, either positive or negative, is offered immediately after the lesson is finished. The authors showed that game performance could be used as a proxy for measuring student classroom performance, on a study performed with second level students learning mathematics.

[30. propose the assessment to be integrated through a quiz, similar in style to "How to be Millionaire". They used as a case study a platform game, where the quiz is inserted between the levels of the game. In this game, a virtual character asks questions and the player has to choose among three options as answers 33 . Feedback is given after the results are submitted. A total of $63 \%$ of the participants involved in the study reported preferring this type of assessment as opposed to taking a "traditional quiz" 30. Moreover, the integrated assessment has not shown to affect the participants enjoyment while playing the game [30].

In Crystal Island [29] a narrative game similar to our game, players are asked to fill quizzes and worksheets through the game. This study does not consider the student's perspective on the integrated assessment and whether this is more effective than when the assessment is not integrated.

There are several aspects in which our research is novel, compared to the previously mentioned ones. As oppose to previous research we are focusing on formalisation and we show how an integrated assessment can also be included as part of the formalisation.

\section{IDS Formal Notion}

This section will first introduce the five usual layers of interactive storytelling Presentation Layer, Conversation layer, Quest Layer, Mission Layer, and Education Layer and afterwards introduces the formalisation of an IDS as FSM. The layered division dawns from the work done by 34 but expands the notion to cover educational remit and designs the actual FSM formalism. A detailed definition of each IDS layer and how they map into the FSM is described below. In doing so, we follow a bottom up approach in which the lower layers are described first. 


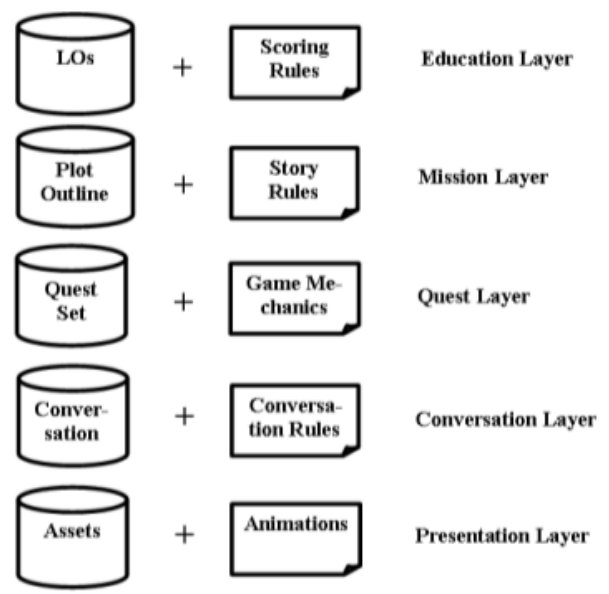

Fig. 1. Interactive Storytelling Layers

\subsection{Presentation Layer}

The Presentation Layer contains the assets/animations needed to deliver the interactive story. It consists of images and animations for characters, rooms, items, in the game, and the motion models that are used to describe how the virtual characters move or behave. They form the interactive story animation function.

Hotspots Hotspots are places where the player can click and invoke an action.

\section{Definition 1. Hotspot}

Let $H$ be a finite non empty set of hotspots, a hotspot, $h \in H$ is defined as a tuple: $h=$ (hotspotImage, action),

where the hotspotImage is a clickable area in an image, and action is the action to be performed as a result (see Definition 7 for more details about game actions).

Item An item is a virtual object that can be collected by the player. The item collection is triggered in response to certain actions performed by the player.

\section{Definition 2. Item}

Let $I$ be a finite non empty set of items, an item, $i \in I$, and is defined as a 3-tuple: $i=$ (itemName, itemImage, hint),

where itemName is a text representing the name of the item, itemImage is a thumbnail image of the item representation, and hint is text to give the player clues about the item (it could be nil). 
Rooms are specific locations in the story. They could be interior rooms, outdoor areas, or sections of a room/outdoor area.

\section{Definition 3. Room}

Let $R$ be a finite non empty set of rooms, a room, $r \in R$ is defined as a 4-tuple: $r=\left(\right.$ roomImage, thumbnailImage, roomName, $\left\{\right.$ hotspot $_{1}, \ldots$, hotspot $\left.\left._{h r}\right\}\right)$, where $\left\{\right.$ hotspot $_{1}, \ldots$, hotspot $\left._{h r}\right\} \in H, h r \in N, h r \geq 0$ defining the number of hotspots in room $r$, roomImage is the location image and thumbanilImage is roomImage in thumbnail. The thumbanilImage can be nil.

Characters Characters are the various story personalities and the player's character. The virtual characters, agents, help the users to accomplish a task [35. In an educational IDS the virtual characters can act as pedagogical agents by helping the player increase his effectiveness by delivering customised advice [36]. This definition is not targeted towards antagonistic characters.

\section{Definition 4. Character}

Let $C$ be a finite non empty set of characters, $C N$ a finite set of conversation nodes (see Definition 5), a character, $c \in C$, is defined as a 5-tuple:

$c=$ (name, characterImage, thumbnailImage, $c n_{c}$, present $\left.C N_{c}\right)$,

where $\left\{c n_{c}\right.$, present $\left.C N_{c}\right\} \in C N$, characterImage is the image representing the character, thumbnailImage is characterImage just thumbnail format.

The player is a special 'player character' $p c \in C$, $p c=\left(\right.$ You, nil, nil, $c n_{Y o u}$, nil).

\subsection{Conversation layer}

The layer on top of the Presentation Layer is the Conversation Layer. In IDS, conversation provides the means of interaction and content presentation [37. This layer consists of Conversation Nodes and Conversation Rules. A Conversation Node $(\mathrm{CN})$ is a line of text or a sentence recited by a character.

The Conversation Rules define which character is saying what, what should happen after the player has read this line of dialogue, and the context in which this happens. For example, a rule could be that a virtual character greets the player at the beginning of the story. Another rule dictates whether the virtual character should greet the player as soon as they meet or wait for the player to initiate conversation. This part has the power to generate adaptive conversations based on the player's previous actions.

\section{Definition 5. Conversation Node}

Let $C N$ be a non empty set of conversation nodes, $C$ a set of characters, then a conversation node, $\mathrm{cn} \in \mathrm{CN}$, is defined as a tuple:

$c n=(c$, Text $)$, where $c \in C$. 
The evaluation of the game LOs could be integrated in IDS as a part of the conversation 38 . In order to do so CNs through which the evaluation questions and feedback were delivered and three conversation rules need to be added as explained in 38. Basically a question will consist of several CNs: the question (each question corresponds to a certain LO), the options the player has to select among and the feedback given (each selected option will trigger a different feedback). The rules are meant to ensure that if the player's prior knowledge is evaluated then the question has to be asked prior to the player being exposed to the $\mathrm{LO}$ while if the player is exposed to the game mechanics the post knowledge is evaluated. The rules also ensure that depending on the selected option the player will get the appropriate feedback. As scoring is also supported by the engine the rules are also meant to ensure that depending on the option selected, the user score will increase/decrease with the appropriate amount. This enables real time feedback for the player.

\subsection{Quest Layer}

The Quest Layer contains the Quest Set and the IDS Mechanics. Quest in the context of this research refers to any story element of the game that requires activation (and later, deactivation) when certain conditions are met. In the context of the IDS FSM it represents a series of events (which could be conversation nodes or actions the player performs) visited according to the transition function and finished when certain conditions are met (Fig. 2). The IDS Mechanics component determines the operations of the game world and deals with the player interactions with the game.

Quest A quest implies achieving a task, exploring a room, collecting evidence, etc. Quests can include other quests, and multiple quests could be opened at the same time.

\section{Definition 6. Quest}

Let $T$ be a set of states, $Q$ a set of states, $t \in T$, is defined as a sequence, $Q$, of states of the IDS FSM starting at $q_{1}$ and finished by reaching state $q_{n}$ following the transition function $\delta$ (see Definition [), $t=\left\{q_{1}, \ldots, q_{n}\right\}, \forall i \in N, \delta: q_{i} \rightarrow$ $q_{i+1}$

where, $Q$ is a set of states, $P$ be a set of game specific conditions, $C$ a set of characters, $R$ a set of rooms, $\left\{q_{1}, q_{n}\right\} \in Q$ are defined as the 4-tuples:

$q_{1}=\left(p_{1}, r_{1}, c_{1}, c n_{1}\right)$,

$q_{n}=\left(p_{n}, r_{n}, c_{n}, c n_{n}\right)$,

where $\left\{p_{1}, p_{n}\right\} \in P, p_{1}$ includes $t$ is open, $p_{n}$ includes $t$ is closed, $r \in R,\left\{c_{1}\right.$, $\left.c_{n}\right\} \in C$, and $\left\{c n_{1}, c n_{n}\right\} \in C N$, and $q_{1}$ is an opening quest state and $q_{n}$ is a closing quest state. 


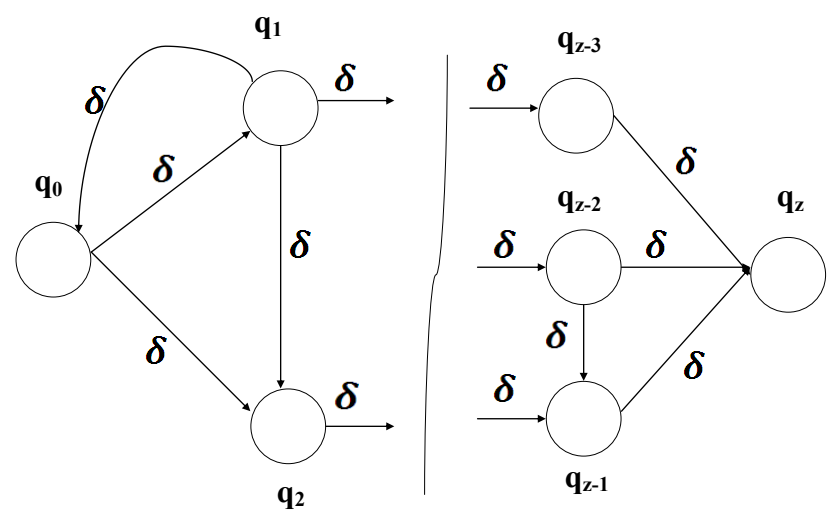

Fig. 2. Finite State-Machine Representation

Action An action is the transition function $\delta$ defined as below:

\section{Definition 7. Transition Function $\delta$ (Action)}

Let $P$ be a set of game specific conditions, $R$ a set of rooms, $C$ a set of characters, and $C N$ a set of conversation nodes. The transition function (game action), $\delta$, is defined as:

$\delta:\left(p, r, c, c n_{0}\right) \rightarrow\left(p^{\prime}, r^{\prime}, c^{\prime},\left\{c n_{1}, \ldots, c n_{i}\right\}\right)$,

where $\{p, p\} \in P, p$ representing a set of pre-conditions for an event to occur, while $p$ ' a set of post-conditions for the event to finish, $\left\{r, r^{\prime}\right\} \in R$, where $r$ can be the same as $r^{\prime},\left\{c, c^{\prime}\right\} \in C$, where $c$ can be the same as $c^{\prime}$, and $\left\{c n_{0}, \ldots\right.$, $\left.c n_{i}\right\} \in C N$ and $\left\{c n_{0}, \ldots, c n_{i}\right\}$ is a set of conversation nodes that follow after $c n_{0}$, typically $i=1$ but it can be empty.

An action could lead to a change in the room the player is in, displaying multiple conversation nodes, etc. A pre-condition to a game action could be that the player clicks on a hotspot. This leads to a change in the FSM states (Fig. 2). Unlike in generic FSM in IDS, the $\delta$ is defined by several predefined actions implementing the IDS mechanics. Several predefined actions were implemented by the IDS engine:

Let $P$ be a set of IDS specific conditions, $\left\{p, p^{\prime}\right\} \in P, p$ representing a set of pre-conditions for an event to occur, and $p$ ' a set of post-conditions, $R$ a set of rooms, $\left\{r, r^{\prime}\right\} \in R, C$ a set of characters, $\left\{c, c^{\prime}\right\} \in C$, and $C N$ a set of nodes, $\left\{c n_{0}, \ldots, c n_{i}\right\} \in C N$. 
- enter room $r$ :

$\delta:\left(p, r, c, c n_{0}\right) \rightarrow\left(p^{\prime}, r^{\prime}, c^{\prime},\left\{c n_{1}, \ldots, c n_{i}\right\}\right)$,

where $r \neq r^{\prime}, p$ and $p^{\prime}$ can be the same state of conditions, $c$ and $c^{\prime}$ can be the same character, $c n_{0}, \ldots, c n_{i}$ can be equal.

- put character $c$ in room $r^{\prime}$ :

$\delta:(p, r, c, n i l) \rightarrow\left(p^{\prime}, r^{\prime}, c, n i l\right)$

- start conversation $c n_{c}$

$\delta:(p, r, c, n i l) \rightarrow\left(p^{\prime}, r^{\prime}, c, c n_{c}\right)$,

- continue conversation $c n_{i-1}$, where the transition function is: $\delta:\left(p, r, c, c n_{i-1}\right) \rightarrow\left(p^{\prime}, r, c^{\prime}, c n_{i}\right)$

- end conversation $\mathrm{cn}_{c}$ :

$\delta:\left(p, r, c, c n_{c}\right) \rightarrow\left(p^{\prime}, r^{\prime}, c, n i l\right)$,

- open quest $t$ :

$\delta:\left(p, r, c, c n_{0}\right) \rightarrow\left(p^{\prime}, r^{\prime}, c^{\prime},\left\{c n_{1}, \ldots, c n_{i}\right\}\right)$,

where $p^{\prime}$ is a set of conditions including t open, $r$, $r$ ' can be the same room, $c$ and $c^{\prime}$ can be the same character, $c n_{0}, \ldots, c n_{i}$ can be equal.

- close quest:

$\delta:\left(p, r, c, c n_{0}\right) \rightarrow\left(p^{\prime}, r^{\prime}, c^{\prime},\left\{c n_{1}, \ldots, c n_{i}\right\}\right)$,

where $p^{\prime}$ is a set of conditions including $\mathrm{t}=$ close, $r, r$ can be the same rooms, $c$ and $c^{\prime}$ can be the same character, $c n_{0}, \ldots, c n_{i}$ can be equal.

\subsection{Mission Layer}

The top level layer contains the overall dramatic outline 34. The story mission is an ultimate quest starting with the game initiation state and finishing when the story is finished by reaching the finish state. It has the highest level of abstraction of the interactive storytelling.

\section{Definition 8. Game Mission - Finite State Engine}

Let $Q$ be a set of states, $P$ be a set of game specific conditions, $R$ a set of rooms, $C$ a set of characters, and CN a set of nodes. An IDS FSM Game Mission is defined as a 4-tuple:

$\left(Q, \delta, q_{0}, q_{z}\right)$

where $\left\{q_{0}, q_{z}\right\} \in Q, q_{0}=\left(p_{0}, r_{0}, c_{0}, c n_{0}\right)$ is the initial state - the start of the mission quest, and $q_{z}=\left(p_{z}, r_{z}, c_{z}, c n_{z}\right)$ is the final state - the end of the mission quest; $\left\{p_{0}, p_{z}\right\} \in P,\left\{r_{0}, r_{z}\right\} \in R,\left\{c_{0}, c_{z}\right\} \in C,\left\{c n_{0}, c n_{z}\right\} \in C N$.

A FSM also defines an alphabet, $\Sigma$. In our case the alphabet is included in the states, rooms, and characters.

\subsection{Educational Layer}

The Education Layer consists of educational content ( $L O s$ that has to be integrated in the game) and Scoring Rules (how much credit would the player get for answering a certain question or performing a certain action in the game). 


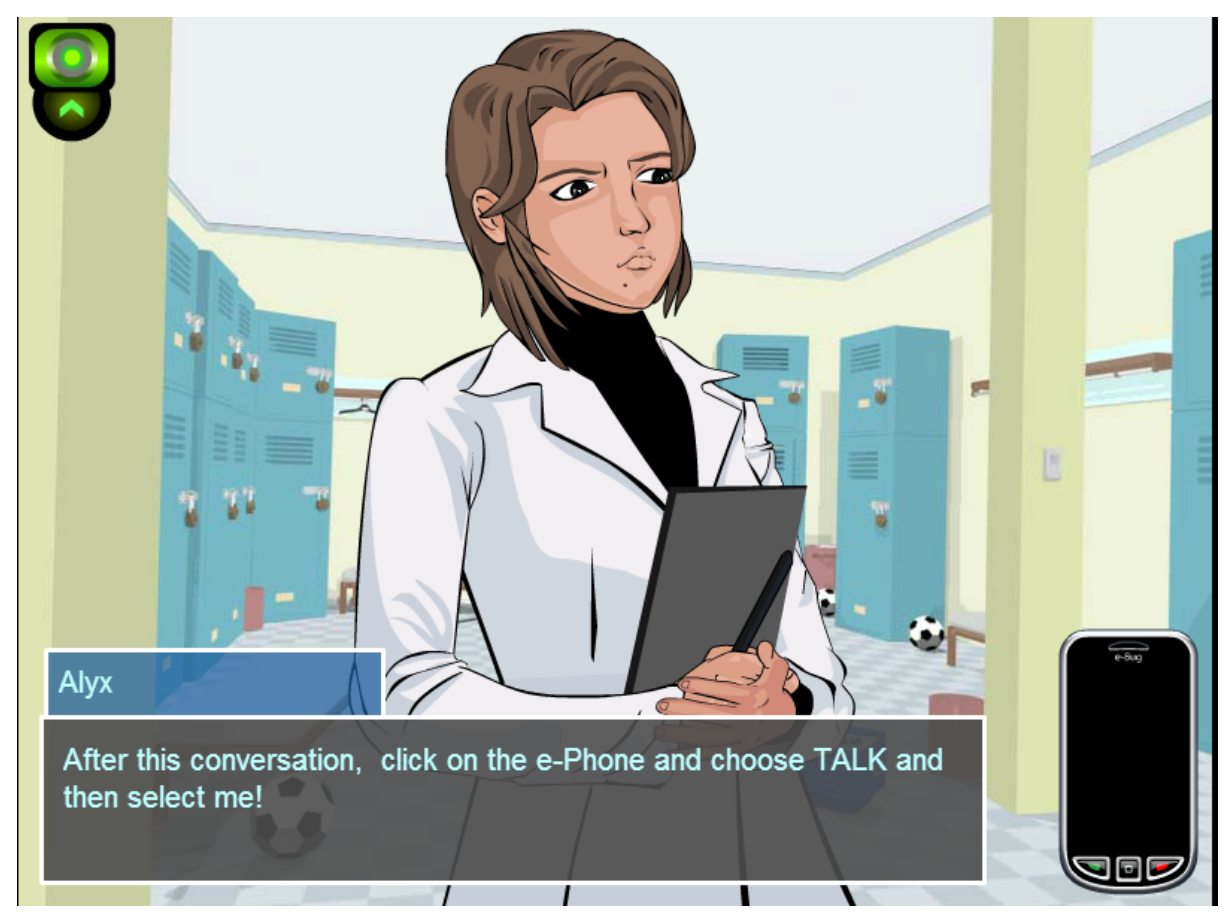

Fig. 3. The Connection between the Authoring Environment, Engine and IDS Generation

The LOs contain a high level description of the LO delivered through the game. For example a LO could be: One should only use antibiotics with a doctor's permission, whereas the CNs contextualise the LOs for the given story. The Scoring Rules consist of rules describing how the LO evaluation, contributes to the player's score.

\section{Game Engine and Authoring Environment}

Based on the proposed formalisation we design and implement a game engine. Authoring the IDS game in the FSM formal notation can be difficult. For example, a definition of Alyx, a character who says Hello and continues saying Welcome to edugames4all is noted according to Definition (4) $A=($ Alyx, image of Alyx, thumbnail image of Alyx, (Alyx, Welcome to edugames), (Alex, Hello)) and requires to express the logical constrains manually. To address this we developed the authoring environment to ease the burden.

With this in mind, the IDS FSM $\delta$ action is implemented in the game engine while the authoring tool lets one define the states of the IDS (Fig. 5). The editor generates files used by the engine to create the IDS. Each set from the IDS FSM is represented by one or more worksheets in the authoring user interface. This 


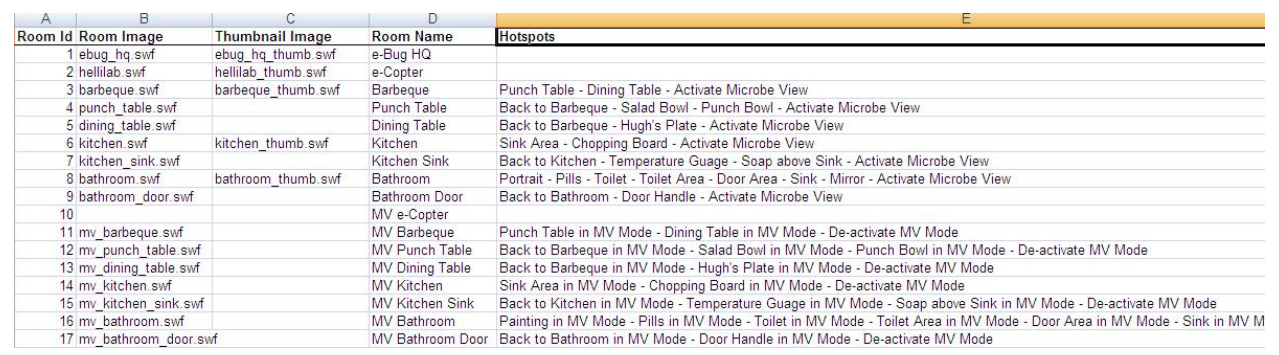

Fig. 4. Rooms Worksheet

allows a more modularised design, the defined semantics becoming more clear and simplifying the game designer's job. The worksheets for each of the layers are:

- Presentation Layer: Rooms, Hotspots, Items, Characters

- Conversation: Conversation Nodes

- Quest: Game Actions, Quests

- Mission: Initial State

Each set is represented in the worksheet with all elements defined as in the IDS FSM. For example, the room has a room image (roomImage), a thumbnail version of the room image (thumbnailRoomImage), the name of the room (roomName) and a set of hotspots $\left(\left\{\right.\right.$ hotspot $_{1}, \ldots$, hotspot hr $\left.\}\right)$. Fig. 4 shows the Rooms worksheet. Each room has its own id, a .swf file containing the room image (the generated IDSes are Flash based), a thumbnail image (the thumbnail image is not mandatory and can be ignored if there is no use for it during game play), the room name, and a set of hotspots that link to the Hotspots worksheet. All the rooms used in the game are defined in this sheet, and when a room is cross-linked from another worksheet it is done by using the id column.

To give some examples on how these are cross-linked, the Hotspots worksheet has references to the actions triggered when a player will click on the hotspot, therefore the action is cross-linked in the Hotspots; the Actions worksheet cross links Rooms, Items and the Conversation Nodes worksheet, in order to specify different actions (e.g. travel between rooms, collecting evidence, starting a new conversation). The main file of the authoring process is the Quest worksheet, which is entirely dependent on the others.

Fig. 5 describes the connection between the engine and the authoring environment. Practically every IDS has different assets, either the images, animations, sound files, etc that are being used to create the IDS. These, together with the IDS flow are described in the authoring editor.

There are several advantages of separating the IDS FSM states (defined in the authoring tool) from the transition function (implemented by the engine). First, designers do not need to modify the engine but can instead focus on defining the user experience in the authoring environment. Second, after the initial investment of building the data-driven engine, any new IDS developed this way requires less effort and investment of time. It is also easier to modify 
since just the files created with the authoring tool need to be changed. Moreover, from an internationalisation and localisation perspective it is more flexible. For example, when translating the dialogue, the translator needs to be given only the file containing the conversation nodes. Third the FSM focuses on the progression rather than on levels, leading to less inconsistency than when the focus is solely on the levels.

The engine and authoring environment were used to create five different IDS based games that can be found at edugames4all. The games we created were constructed with the aim of creating awareness about issues related to health in an enjoyable manner. The translation and localisation were done using the authoring tool by public health specialists and a microbiologist from the country of the language in which the story was translated. None of the public health specialists or microbiologists had any computing background. The games and the translations were created entirely using the authoring tool and the generated files were given to the game engine at run-time to generate the games.

The games are part of the edugames4all project which has followed e-Bug, the project under which some of the games were originally created. e-Bug was an European project in which researchers and practitioners from 18 European countries were partially funded under The Directorate-General for Health and Consumers (DG SANCO) of the European Commission [39. The project aim was to develop and disseminate educational resources that teachers would use to teach junior and senior school children [40. With this aim an EU wide survey was distributed to assess children knowledge of hygiene to set the LOs at a common denominator 41. Two types of games were created from LOs found as a result of the survey: a platform game for junior kids 42] and an IDS based game for 13-15 years old [21].

\section{Challenges and Proposed Solutions}

As the previous sections addressed the formalisation and authoring of IDS based games, this section presents some of the challenges encountered when the games were already implemented and how we addressed them. These are grouped in three sections addressing the drop-out rate, game usability assessment and educational content evaluation.

\subsection{Drop-out Rate}

Drop out rates are a known issue for computer games as reported by academic studies of educational games 43 as well as in industry settings 44 . In our case, IDS based games were played by over 60.000 users in 2010 [45. However in all cases when the games were introduced in the classroom it was noticed that there was a dropout in the number of students that finish playing relatively to those that started [46. One of the problems was that not all the students played a similar game before. A text tutorial was integrated in the game but was rarely consulted by the children. To help alleviate this problem, another mission called 


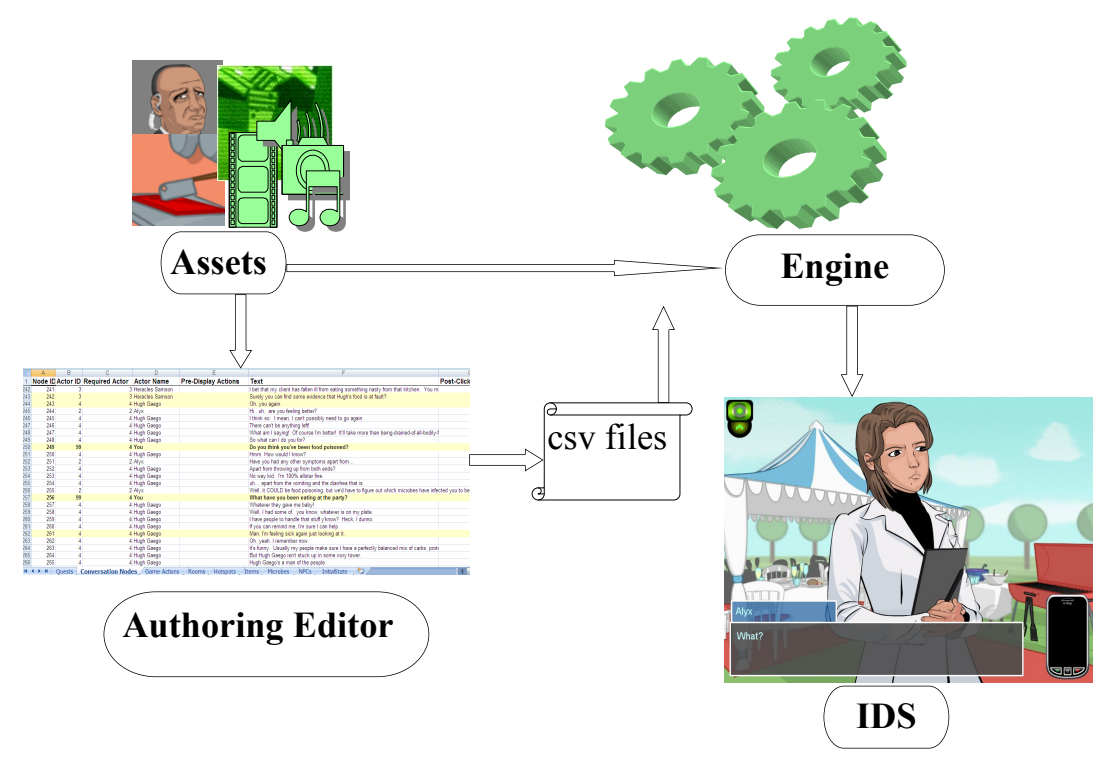

Fig. 5. The Virtual Character Providing Guidance

Training Mission [46], was implemented to teach the students the mechanics of the game. The mission is shorter than the other missions and a lot of guidance is provided by the non-player character. The player is shown how to use travel between different locations, how to collect and analyse evidence, and the usage of microvision, elements pertinent to solving the mystery in the rest of the missions (Fig. 3).

The usability of this mission was initially tested with 49 students at a summer school and assessed through five open questions targeting the mission graphics, easiness to follow directions through the game, willingness to continue playing and improvements to be made [46. Most of the participants liked the graphics, found the Training Mission easy to play and they wanted to continue playing. A second study of the Training Mission took place with 15 students divided into two groups. One group played one of the missions without the Training Mission beforehand and the other one played the Training Mission and afterward a mission of the game. The researcher taking part in the study observed the children and reported that the children who played the Training Mission beforehand asked less for help and more students completed the game in this scenario as opposed to the previous one [46]. A second evaluation was performed to determine whether playing the training mission before playing the other missions affects the usability of the other missions [47. Although the results showed that when playing the training mission the perceived usability of the other missions 
is increased, no statistically significant difference was obtained except for the fact that the children perceived the missions as less awkward when they play the training mission beforehand.

\subsection{Game Usability Assessment}

System Usability Scale (SUS) [48] is a questionnaire that has been extensively used in testing usability. Because the SUS questionnaire has been designed for analysing systems in general, the questionnaire was adapted to our situation by changing the word "system" to "game" and "use" with "play" (see Table 1 for the full modified questionnaire). Replacing similar terms in a SUS questionnaire has been found not to affect its reliability and validity [49, [50].

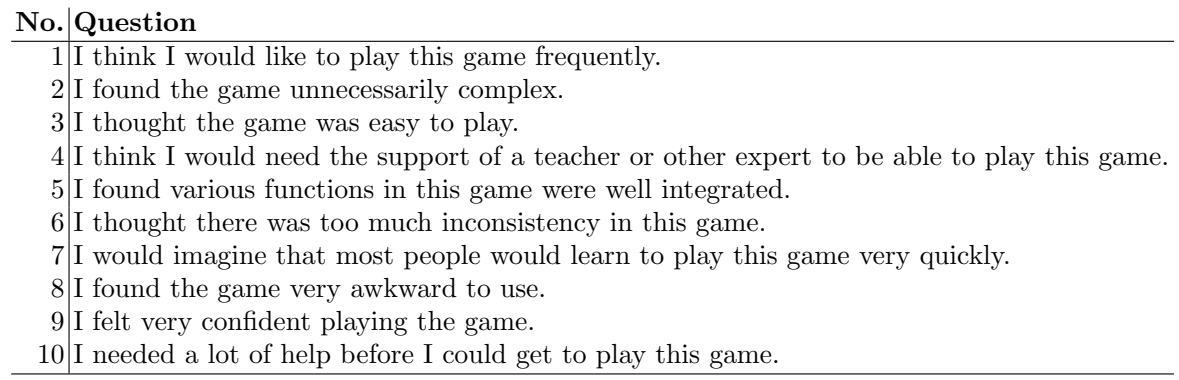

Table 1. SUS Questionnaire Adapted for the Game

SUS is a short survey, consisting of 10 questions. It indicates the usability of a system in general, and a game in this particular case, on a scale from 0 to 100, where 100 is the best score that can be obtained. For each question the subject has to choose an option on a 5 point Likert scale to indicate the degree of agreement or disagreement with the statement in the question. In this case 1 stands for Strongly Disagree and 5 stands to Strongly Agree. To compute the SUS score, the following steps are required. For the odd questions one has to subtract 1 from the option the subject chooses on the Likert scale, while for the even questions the chosen scale position is subtracted from 5 . In this way each of the questions contributes with a value from 0 to 4 to the final score. The sum of the results computed in this way is multiplied by 2.5 and divided by the number of subjects. The number obtained in this way is the SUS score. The SUS score for our game is 66.78 out of 100 which is considered to be a Good usability score 49 .

As SUS is just a measure of quantifying the usability and not a diagnostics tool, several other questions were added in the survey to determine areas for improving the game and when the game was played in schools, focus groups with the children and the teachers took place to further drill down on the issues that need improvement (some of the teachers were eager to play the games themselves and they also provided feedback). 


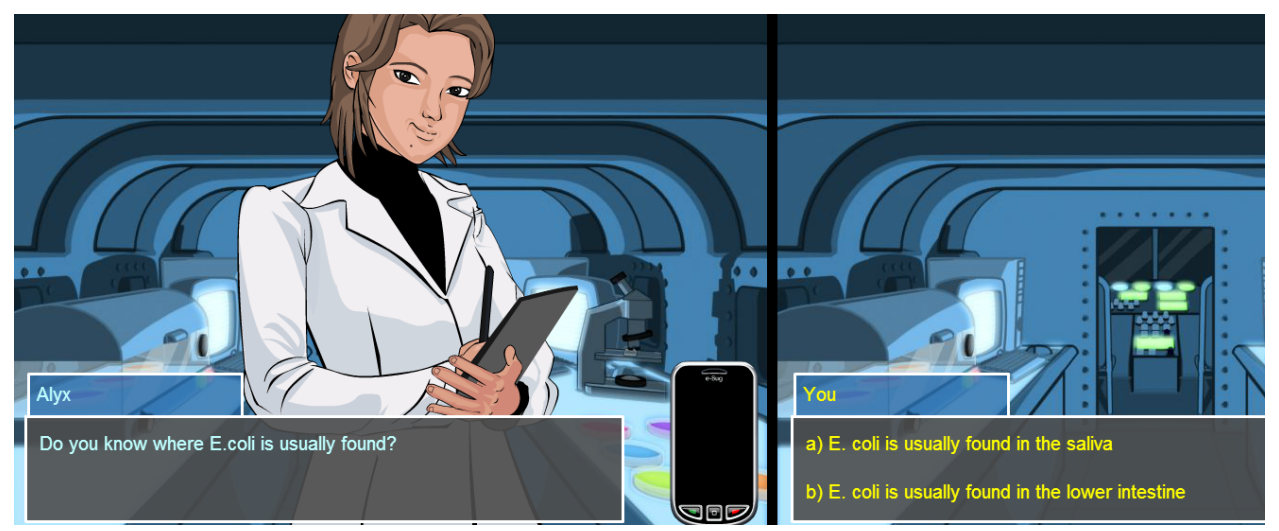

Fig. 6. Pre-knowledge evaluation

In the survey the players were asked about having difficulties on specific parts of the game and most of them reported on not having any. When asked what they liked most about this game, the players said: the educational nature of the game, characters, that it was funny, intuitive etc. Among the parts disliked was the fact that there was too much text to read, the slow curve to learn the game, the story etc. There were some comments to which the players answers contradict, some subjects disliking that the game is too short while others consider it is too long. When asked in the survey, as a separate question if they consider the length of the game appropriate, $85 \%$ considered it was the appropriate length.

\subsection{Assessing Educational Potential}

Assessment of educational content integrated in the game is unpopular with game users and especially children. Seamless evaluation was implemented to overcome this issue. The questionnaire assessing the knowledge assessment was basically integrated in the IDS story in such a way that most of the players did not realise they were being evaluated [38. The questions are asked at different stages of the game attention being paid that a first round of questions is asked before the player is exposed to the learning objectives and another one after the player is exposed to the game mechanics. For example, we integrated this questionnaire in one of the missions, having one of the virtual characters ask the player questions during the investigation - questions that will be part of the pre-test of the players knowledge, (Fig. 6) and the post-test questions were asked when the player returns to the agency during the debriefing (Fig. 7). This allowed us to assess the players knowledge without affecting the game experience and also without asking the children to complete a questionnaire when playing the game. 


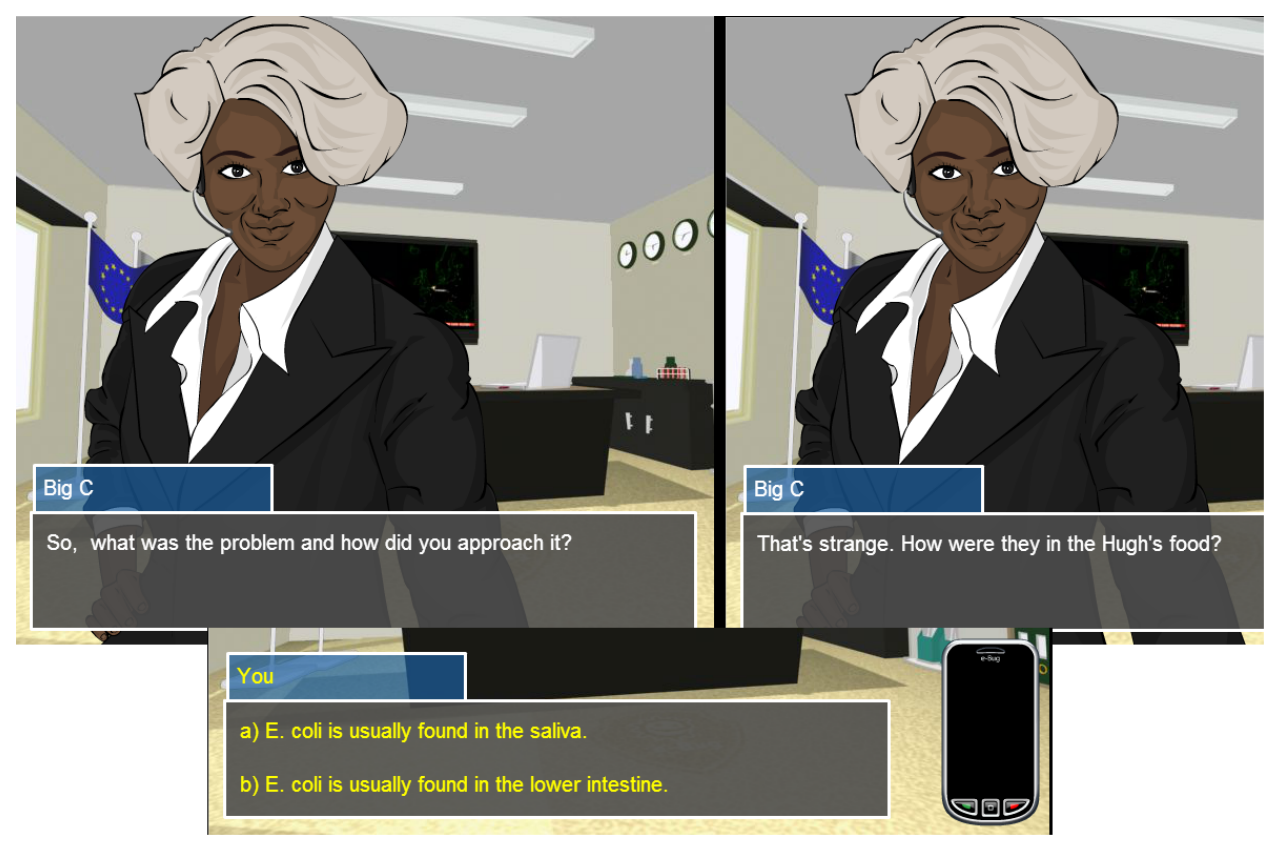

Fig. 7. Post-knowledge evaluation

\section{Discussions and Work in Progress}

Although a user centred design was followed through the game's design and implementation, where teachers and students were consulted from the initial stages of the project and during the implementation to iteratively improve the games [51] a high drop out rate was noticed. One reason for the drop out rate were the students low reading skills. Due to the high textual content of the game, sometimes the children found it tiring reading through the game. We are currently working on also having a version of the game that will contain more audio, the characters being able to speak to the user through voice rather than through text as it is currently implemented.

We are planning on adding gamification elements to the website hosting the games, such as league tables with the players that get bigger scores in the game. We want the children to be able to easily share their score in the game with others. We hope that this will also help with the retention in playing the games and that the children will attempt to improve their scores, scores that are computed by taking into account the LOs evaluation. Moreover we are currently working on having the games available on mobile phones as mobile apps.

Another challenge that is undergoing is setting the right level of complexity in the game not only by adjusting the player knowledge but also the game mechanics. We want to determine during the game play if the player needs help without him requesting it. Player knowledge is difficult to assess in an online 
game, played on-line by children from different countries. We addressed this by assessing the LOs across European countries to be able to cover the core LOs. Moreover, the game has some level of adaptation based on the previous user knowledge (e.g. depending on the LOs displayed in a certain part of the game, the LOs in some other part of the game may or may not be displayed). Currently we are working on improving the knowledge adaptation.

\section{Conclusions}

IDS based games have been growing in popularity both in entertainment but also as a means of delivering educational content in an enjoyable manner to children. As game story writers do not always come from computing backgrounds, there is a need for a user-friendly authoring tool to help in alleviating the authoring process. In this paper we presented how IDS can be formalised using FSM and a game engine was implemented based on this formalisation. The game engine takes input files generated by an authoring tool. This engine and authoring tool were used in implementing several IDS based games as part of the e-bug/edugames4all project with a number of authors in different countries who used the tool for localisation and translation. We also presented a standard solution to three common game play challenges encountered when evaluating the games.

\section{Acknowledgment}

The authors would like to thank e-Bug project members and DG SANCO for providing funding for this EU project. We would also like to thank digital artists Nancy Lai and Sandy Beveridge who created the project artwork.

\section{References}

1. Park, N., Min Lee, K., Annie Jin, S.A., Kang, S.: Effects of pre-game stories on feelings of presence and evaluation of computer games. International journal of human-computer studies 68(11) (2010) 822-833

2. HodHod, R.A., Cairns, P.A., Kudenko, D.: Innovative integrated architecture for educational games: Challenges and merits. T. Edutainment 5 (2011) 1-34

3. Lee, S.Y., Mott, B.W., Lester, J.C.: Director agent intervention strategies for interactive narrative environments. In Si, M., Thue, D., Andre, E., Lester, J.C., Tanenbaum, J., Zammitto, V., eds.: ICIDS. Volume 7069 of Lecture Notes in Computer Science., Springer (2011) 140-151

4. Smith, G., Anderson, R., Kopleck, B., Lindblad, Z., Scott, L., Wardell, A., Whitehead, J., Mateas, M.: Situating quests: Design patterns for quest and level design in role-playing games. In Si, M., Thue, D., Andre, E., Lester, J.C., Tanenbaum, J., Zammitto, V., eds.: ICIDS. Volume 7069 of Lecture Notes in Computer Science., Springer (2011) 326-329 
5. Lewis, C., Whitehead, J.: The whats and the whys of games and software engineering. In: Proceedings of the 1st International Workshop on Games and Software Engineering, New York, NY, USA, ACM (2011) 1-4

6. Cavazza, M., Champagnat, R., Leonardi, R.: The iris network of excellence: Future directions in interactive storytelling. In: Proceedings of the 2nd Joint International Conference on Interactive Digital Storytelling: Interactive Storytelling. ICIDS '09, Berlin, Heidelberg, Springer-Verlag (2009) 8-13

7. Jantke, K.P.: Logical formalization and reasoning for computerized interactive storytelling. In: Progress in Informatics and Computing (PIC), 2010 IEEE International Conference on. Volume 2., IEEE (2010) 851-857

8. Magy, S.E.N., David, M., Tony, M.: Experiencing interactive narrative: A qualitative analysis of faade. Entertainment Computing 4(1) (2013) 39-52

9. Peinado, F., Gerves, P.: Transferring game mastering laws to interactive digital storytelling. In Gobel, S., Spierling, U., Hoffmann, A., Iurgel, I., Schneider, O., Dechau, J., Feix, A., eds.: TIDSE. Volume 3105 of Lecture Notes in Computer Science., Springer (2004) 48-54

10. Ong, T., Leggett, J.J.: A genetic algorithm approach to interactive narrative generation. In: Proceedings of the fifteenth ACM conference on Hypertext and hypermedia. HYPERTEXT '04, New York, NY, USA, ACM (2004) 181-182

11. Cai, Y., Miao, C., Tan, A.H., Shen, Z.: Fuzzy cognitive goal net for interactive storytelling plot design. In: Proceedings of the 2006 ACM SIGCHI international conference on Advances in computer entertainment technology, New York, NY, USA, ACM (2006)

12. Mateas, M., Stern, A.: Natural language understanding in facade: Surface-text processing. In Gobel, S., Spierling, U., Hoffmann, A., Iurgel, I., Schneider, O., Dechau, J., Feix, A., eds.: TIDSE. Volume 3105 of Lecture Notes in Computer Science., Springer (2004) 3-13

13. Cavazza, M., Lugrin, J.L., Pizzi, D., Charles, F.: Madame bovary on the holodeck: immersive interactive storytelling. In: Proceedings of the 15 th international conference on Multimedia. MULTIMEDIA '07, New York, NY, USA, ACM (2007) $651-660$

14. Mahmud, N., Papadopoulos, Y., Walker, M.: A translation of state machines to temporal fault trees. In: Proceedings of the 2010 International Conference on Dependable Systems and Networks Workshops (DSN-W). DSNW' 10, Washington, DC, USA, IEEE Computer Society (2010) 45-51

15. Dang, K.D., Champagnat, R., Augeraud, M.: Modeling of interactive storytelling and validation of scenario by means of linear logic. In: Interactive Storytelling. Springer (2010) 153-164

16. Porteous, J., Cavazza, M.: Controlling narrative generation with planning trajectories: the role of constraints. In: Interactive Storytelling. Springer (2009) 234-245

17. Gerevini, A., Long, D.: Plan constraints and preferences in pddl3. The Language of the Fifth International Planning Competition. Tech. Rep. Technical Report, Department of Electronics for Automation, University of Brescia, Italy 75 (2005)

18. Di Blas, N., Paolini, P.: Digital storytelling and educational benefits: Evidences from a large-scale project. In: Transactions on Edutainment X. Springer (2013) 83-101

19. Lim, T., Louchart, S., Suttie, N., Hauge, J.B., Stanescu, I.A., Ortiz, I.M., MorenoGer, P., Bellotti, F., Carvalho, M.B., Earp, J., et al.: Narrative serious game mechanics (nsgm)-insights into the narrative-pedagogical mechanism. In: Games for Training, Education, Health and Sports. Springer (2014) 23-34 
20. Kapp, K.M.: The gamification of learning and instruction: game-based methods and strategies for training and education. John Wiley \& Sons (2012)

21. Molnar, A., Kostkova, P.: 13 learning through interactive digital narratives. Interactive Digital Narrative: History, Theory and Practice (2015) 200-210

22. Paliokas, I., Arapidis, C., Mpimpitsos, M.: Game based early programming education: the more you play, the more you learn. In: Transactions on Edutainment IX. Springer (2013) 115-131

23. Padilla-Zea, N., Gutiérrez, F.L., López-Arcos, J.R., Abad-Arranz, A., Paderewski, P.: Modeling storytelling to be used in educational video games. Computers in Human Behavior 31 (2014) 461-474

24. Liu, S., Ding, W.: An approach to evaluation component design in building serious game. In: Learning by Playing. Game-based Education System Design and Development. Springer (2009) 141-148

25. Wendel, V., Göbel, S., Steinmetz, R.: Seamless learning in serious games-how to improve seamless learning-content integration in serious games. In: CSEDU. (2011) 219-224

26. Torrente, J., Moreno-Ger, P., Fernández-Manjón, B., Del Blanco, Á.: Game-like simulations for online adaptive learning: A case study. In: Learning by Playing. Game-based Education System Design and Development. Springer (2009) 162-173

27. McAlpine, M., van der Zanden, L., Harris, V., Authority, S.Q.: Using games based technology in formal assessment of learning. In: Proceedings of the 4th European Conference on Games-Based Learning: The Danish School of Education, Aarhus University, Copenhagen, Denmark, 21-22 October 2010, Academic Conferences Limited (2010) 242

28. Molnar, A., Virseda, J., FriAs-MArtinez, V.: Insights from educamovil: Involving teachers in creating educational content for mobile learning games. Journal of Interactive Learning Research 26(2) (2015) 209-221

29. Spires, H.A., Rowe, J.P., Mott, B.W., Lester, J.C.: Problem solving and gamebased learning: Effects of middle grade students' hypothesis testing strategies on learning outcomes. Journal of Educational Computing Research 44(4) (2011) 453472

30. Kostkova, P., Molnar, A.: Educational games for creating awareness about health issues: The case of educational content evaluation integrated in the game. In: Proceedings of the Med 2.0

31. Higgins, R., Hartley, P., Skelton, A.: The conscientious consumer: reconsidering the role of assessment feedback in student learning. Studies in higher education 27(1) (2002) 53-64

32. Persky, A.M., Pollack, G.M.: Using answer-until-correct examinations to provide immediate feedback to students in a pharmacokinetics course. American journal of pharmaceutical education 72(4) (2008)

33. Molnar, A., Kostkova, P.: On effective integration of educational content in serious games: Text vs. game mechanics. In: Advanced Learning Technologies (ICALT), 2013 IEEE 13th International Conference on. (2013) 299-303

34. Spierling, U.: Interactive digital storytelling: Towards a hybrid conceptual approach. In: DIGRA Conf. (2005)

35. Sklar, E.: Agents for education: when too much intelligence is a bad thing. In: Proceedings of the second international joint conference on Autonomous agents and multiagent systems, ACM (2003) 1118-1119

36. Conati, C., Zhao, X.: Building and evaluating an intelligent pedagogical agent to improve the effectiveness of an educational game. In: Proceedings of the 9th international conference on Intelligent user interfaces, ACM (2004) 6-13 
37. Spierling, U.: Introducing interactive story creators to conversation modelling. In: Proceedings of the 8th International Conference on Advances in Computer Entertainment Technology. ACE '11, New York, NY, USA, ACM (2011) 38:1-38:8

38. Molnar, A., Kostkova, P.: Seamless evaluation integration into ids educational games. In: FDG. (2013) 322-329

39. McNulty, C.A., Lecky, D.M., Farrell, D., Kostkova, P., Adriaenssens, N., Herotová, T.K., Holt, J., Touboul, P., Merakou, K., Koncan, R., et al.: Overview of e-bug: an antibiotic and hygiene educational resource for schools. Journal of antimicrobial chemotherapy 66(suppl 5) (2011) v3-v12

40. Lecky, D.M., McNulty, C.A., Adriaenssens, N., Herotová, T.K., Holt, J., Kostkova, P., Merakou, K., Koncan, R., Olczak-Pienkowska, A., Avô, A.B., et al.: Development of an educational resource on microbes, hygiene and prudent antibiotic use for junior and senior school children. Journal of antimicrobial chemotherapy 66(suppl 5) (2011) v23-v31

41. Lecky, D.M., McNulty, C.A., Adriaenssens, N., Herotová, T.K., Holt, J., Touboul, P., Merakou, K., Koncan, R., Olczak-Pienkowska, A., Avô, A.B., et al.: What are school children in europe being taught about hygiene and antibiotic use? Journal of antimicrobial chemotherapy 66(suppl 5) (2011) v13-v21

42. Farrell, D., Kostkova, P., Weinberg, J., Lazareck, L., Weerasinghe, D., Lecky, D.M., McNulty, C.A.: Computer games to teach hygiene: an evaluation of the e-bug junior game. Journal of antimicrobial chemotherapy 66(suppl 5) (2011) v39-v44

43. Frias-Martinez, V., Virseda, J., Gomero, A.: Mobilizing education: evaluation of a mobile learning tool in a low-income school. In: Proceedings of the 14th international conference on Human-computer interaction with mobile devices and services, ACM (2012) 441-450

44. Snow, B.: Why most people don't finish video games. Online publication in CNN, August 17 (2011) 2011

45. Weerasinghe, D., Lazareck, L., Kostkova, P., Farrell, D.: Evaluation of popularity of multi-lingual educational web games: do all children speak english? In: 3rd International ICST Conference on Electronic Healthcare for the 21st century. (2010)

46. Molnar, A., Kostkova, P.: If you build it would they play? challenges and solutions in adopting health games for children. In: Proceedings of ACM SIGCHI Conference on Human Factors in Computing Systems, Let's talk about Failures: Why was the Game for Children not a Success. (2013)

47. Molnar, A., Kostkova, P.: Gaming to master the game-game usability and game mechanics. In: Serious Games and Applications for Health (SeGAH), 2014 IEEE 3rd International Conference on, IEEE (2014) 1-7

48. Jordan, P.W., Thomas, B., McClelland, I.L., Weerdmeester, B.A., eds. In: SUS: A quick and dirty usability scale. Taylor and Francis (1996) 189-194

49. Bangor, A., Kortum, P.T., Miller, J.T.: An empirical evaluation of the system usability scale. Intl. Journal of Human-Computer Interaction 24(6) (2008) 574594

50. Lewis, J.R., Sauro, J.: The factor structure of the system usability scale. In: Human Centered Design. Springer (2009) 94-103

51. Lazareck, L., Farrell, D., Kostkova, P., Lecky, D., McNulty, C., Weerasinghe, D.: Learning by gaming-evaluation of an online game for children. In: Engineering in Medicine and Biology Society (EMBC), 2010 Annual International Conference of the IEEE, IEEE (2010) 2951-2954 